DeC 13Th 2007: ACCEPTED For PUblication in ApJ LetTers

Preprint typeset using LTEX style emulateapj v. 08/22/09

\title{
WHERE CENTAURUS A GETS ITS X-RAY KNOTTINESS
}

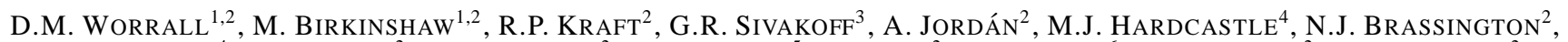

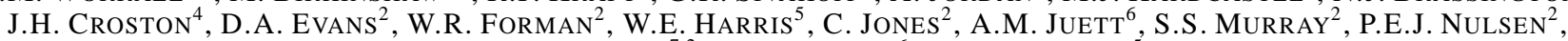 \\ S. RAYCHAUDhURY ${ }^{7,2}$, C.L. SARAZIN ${ }^{6}$, K.A. WOODLEY ${ }^{5}$ \\ Dec 13th 2007: Accepted for publication in ApJ Letters
}

\begin{abstract}
We report an X-ray spectral study of the transverse structure of the Centaurus A jet using new data from the Chandra Cen A Very Large Project. We find that the spectrum steepens with increasing distance from the jet axis, and that this steepening can be attributed to a change in the average spectrum of the knotty emission. Such a trend is unexpected if the knots are predominantly a surface feature residing in a shear layer between faster and slower flows. We suggest that the spectral steepening of the knot emission as a function of distance from the jet axis is due to knot migration, implying a component of transverse motion of knots within the flow.

Subject headings: galaxies: active — galaxies: individual (Centaurus A, NGC 5128) — galaxies: jets — X-rays: galaxies
\end{abstract}

\section{INTRODUCTION}

In relatively few extragalactic radio jets can the kinematics of the flow on kpc scales be studied with any degree of certainty. Most arguments are indirect, relying either on beaming statistics for samples (e.g., Hardcastle et al. 2003a) or, for powerful jets oriented along the line of sight, on models relying on an assumed inverse-Compton origin for the X-ray emission (e.g., Schwartz et al. 2006). For the few low-power radio galaxies with heavily studied, straight, radio jets and counterjets, kinematic models have been constructed based on the jet-counterjet asymmetry (e.g., Canvin et al. 2005). These models have supported jet deceleration through mass entrainment, and ongoing work has already shown consistency between the density and pressure model inferred from the jet and the properties of the external X-ray-emitting gas for 3C 31 (Laing \& Bridle 2002).

In models of mass entrainment, the outer parts ("sheath") are decelerated before the inner ("spine"), consistent with a range of observational evidence at radio frequencies (Laing 1996). Applied to more central regions, the consequence that emission from a slower sheath becomes relatively more important in jets at larger angle to the line of sight then resolves difficulties in models that unify BL Lac objects with low power radio galaxies (e.g., Chiaberge et al. 2000).

Chandra has not only allowed confirmation that X-ray synchrotron emission in low-power jets is common (Worrall et al. 2001), but has also resolved transverse structure in the nearest and brightest sources. A particularly interesting example is NGC 315 (Worrall et al. 2007). Here the diffuse emission contains a knotty structure in the radio and X-ray that ap-

\footnotetext{
${ }^{1}$ Department of Physics, University of Bristol, Tyndall Avenue, Bristol BS8 1TL, UK

${ }^{2}$ Harvard-Smithsonian Center for Astrophysics, 60 Garden Street, Cambridge, MA 02138

3 Department of Astronomy, The Ohio State University, 4055 McPherson Laboratory, 140 W. 18th Avenue, Columbus, OH 43210

${ }^{4}$ School of Physics, Astronomy \& Mathematics, University of Hertfordshire, College Lane, Hatfield AL10 9AB, UK

${ }^{5}$ Department of Physics and Astronomy, McMaster University, Hamilton, ON L8S 4M1, Canada

6 Department of Astronomy, University of Virginia, P. O. Box 400325, Charlottesville, VA 22904

7 School of Physics and Astronomy, University of Birmingham, Edgbaston, Birmingham B15 2TT, UK
}

pears to describe an oscillatory filament. Although the structure could be the result of a chance superposition of nonaxisymmetric knots, the level of coherence led us to suggest that the knots might be predominantly a surface feature residing in the shear layer between the spine and sheath. If this latter interpretation is correct, we might expect the X-ray spectra of the knots to be similar across the transverse width of the jet.

In the case of NGC 315 the distinct knotty emission is only about $10 \%$ of the total emission in X-rays and radio along the $\sim 2.5 \mathrm{kpc}$ of projected jet length over which it is detected, and with a source distance of $\sim 70 \mathrm{Mpc}$ the observations did not allow us to measure separate X-ray spectra for the knots and diffuse emission. At $3.7 \mathrm{Mpc}^{8}$ (1 arcmin is $\sim 1.1 \mathrm{kpc}$ projected length), Centaurus $\mathrm{A}$ is a much closer example of a low-power radio galaxy whose inner jet shows bright resolved X-ray knots and diffuse emission over a similar projected length scale to NGC 315 (e.g. Kraft et al. 2002). Here we report an X-ray spectral study of the transverse structure of the jet, and in particular we investigate whether the Cen A knots are likely to occupy a shear layer between faster inner and slower outer flows.

\section{OBSERVATIONS \& ANALYSIS}

The Chandra data used here are from the Cen A Very Large Project (Cen A-VLP), comprising six deep ( $\sim 100 \mathrm{ks})$ exposures (OBSIDs 7797, 7798, 7799, 7800, 8489, 8490) of the front-illuminated CCD chips of the Advanced CCD Imaging Spectrometer (ACIS) made between 2007 March 7 and May 30. Standard methods ${ }^{9}$ have been followed for data reprocessing and grade selection, and small astrometric corrections were made to register the six exposures to a common frame using detected point sources (Hardcastle et al. 2007; Jordán et al. 2007). The 0.8-3 keV merged image of the inner $\sim 4^{\prime}$ of the X-ray jet is shown in Figure 1

We are interested here in the jet after it has become radiatively bright in both radio and X-ray (more than $21^{\prime \prime}$ from the core, and after knots $\mathrm{AX} 1 \mathrm{~A}, \mathrm{AX} 1 \mathrm{C}$ in the terminology of Hardcastle et al. 2003b) until its major decline relative to the radio (at $\sim 141^{\prime \prime}$ ). Motivated by NGC 315 , where the fast spine is approximately $60 \%$ of the jet width, we have defined

\footnotetext{
8 The average of five distance indicators, see $\S 6$ in Ferrarese et al. (2007)

${ }^{9} \mathrm{http}: / /$ asc.harvard.edu/ciao/
} 


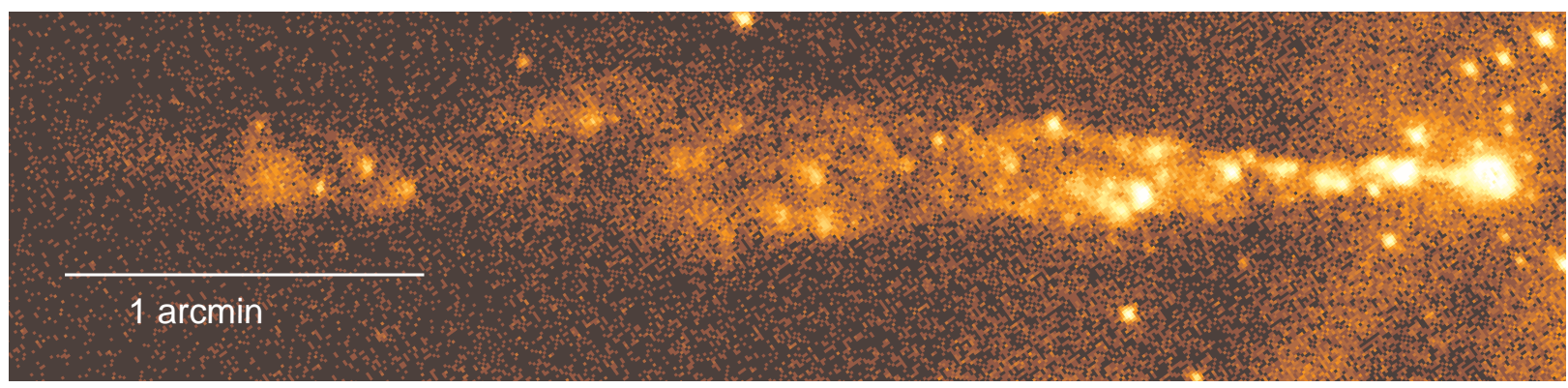

FIG. 1.- 0.8-3-keV unsmoothed rotated image of the six Chandra exposures comprising the Cen A-VLP, with $0.492 \times 0.492 \operatorname{arcsec}^{2}$ pixels.

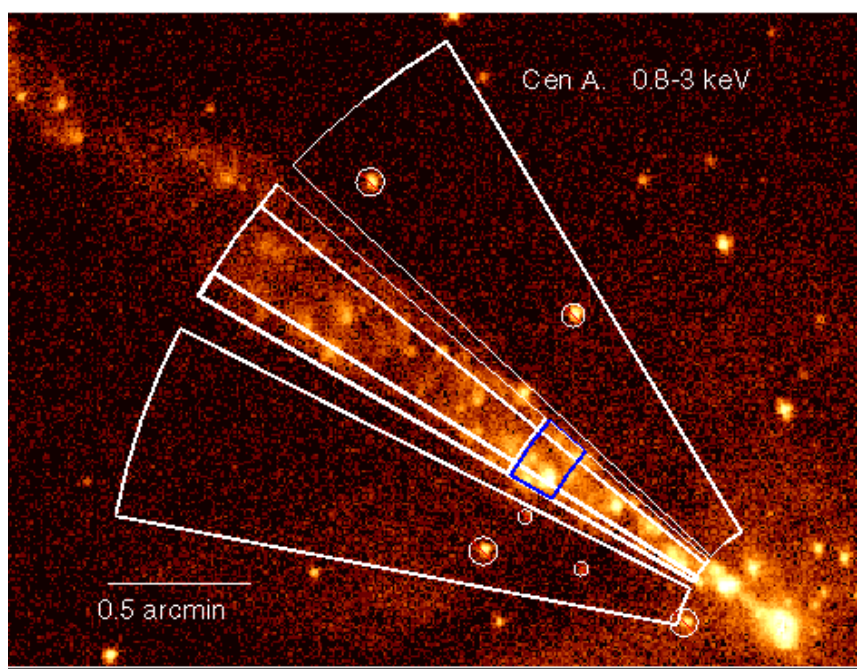

FIG. 2. - Image from Fig. 11 with regions superposed, and rotated to the sky orientation. The regions, called the spine, sheath and background, working outwards from the jet axis, are pie slices from a base position of R.A. $=13^{\mathrm{h}} 25^{\mathrm{m}} 26.98$, Dec. $=-43^{\circ} 01^{\prime} 14^{\prime \prime} .06$ (not the core) running from position angle (pa) $51.2-57.8$ for the spine, pa $49^{\circ}-51.2$ and $57.8-60^{\circ}$ for the sheath, and pa $31^{\circ}-47^{\circ}$ and $63^{\circ}-79^{\circ}$ for the background with the exclusion regions shown. From the core the inner spine and sheath regions are at a distance of $21^{\prime \prime}$ to $66^{\prime \prime}$, and the outer region runs from $66^{\prime \prime}$ to $141^{\prime \prime}$. We call the region marked in blue, near the outer extent of the inner region, the "bright" region.

regions shown in Figure 2. While not wishing to suggest $a$ priori that the Cen A jet does have a slower sheath and faster spine, for shorthand we call these regions the spine, sheath and background, working outwards from the jet axis. Compact sources excluded from the background regions are also marked. A division of the spine and sheath into two regions with length down the jet is shown. We refer to these as the inner and outer regions, and the boundary was chosen so that there is a comparable number of total counts in each region.

The jet is positioned on a single ACIS I chip in all six exposures. The boundary between the outer and inner regions lies between 1.4 and 4.0 arcmin off axis depending on OBSID, giving a point-spread-function (PSF) half-powerdiameter (HPD) at this location of better than about 1 arcsec at the $\sim 1.5-\mathrm{keV}$ energy of maximum count rate per keV. The readout streak from the Cen A core runs close to the SE sheath region in OBSID 7797, and so we exclude this observation in spectral fitting to the SE sheath and inner spine. We extract data and responses for identical sky regions in the different OBSIDs, and we fit the same model simultaneously to the data sets. Spectra are binned to a minimum of 30 counts per bin so that the $\chi^{2}$ statistic can be applied.

In this work we are searching for statistical trends in spectral index in knotty and diffuse emission transverse to the jet axis. Knots are identified as bright enhancements, but we believe them to be composed of multiple distinct physical structures, each potentially with a different emission spectrum. The precise definition of knots is therefore not crucial, and we have adopted the previously published lists of Kraft et al. (2002) and Kataoka et al. (2006), which combined give 31 knots within our total spine and sheath regions. The knots were examined visually, and small centroid shifts (within uncertainties) were made to give best alignment in the new data. A circle of nominal radius 2.4 arcsec was used to mask each knot. Four additional weak but compact enhancements seen in the new data were also masked as knots with circles of radii 1.2 arcsec. Because of our interest in statistical trends, for knots that overlap regions we divide the counts according to where they were detected. When extracting spectra of the knots the diffuse (residual) emission in the corresponding region is used as background. The outer sectors shown in Figure 2 provide background for the diffuse emission.

All our spectral models are single-component power laws with free hydrogen column density, $N_{\mathrm{H}}$, over the energy range $0.4-10 \mathrm{keV}$. Intrinsic $N_{\mathrm{H}}$ in Cen A over and above the Galactic value of $8.4 \times 10^{20} \mathrm{~cm}^{-2}$ (Dickey \& Lockman 1990) causes reductions in the count rate in soft X-ray images, consistent with the dust lanes that cross the jet roughly transverse to its axis. Since our regions extend hundreds of pc along the jet, we are combining data with different intrinsic $N_{\mathrm{H}}$. This is expected to give a bias towards finding slightly flatter power-law slopes than the true slope if the jet emission is characterized by a single spectrum over its length. However, the gradients in $N_{\mathrm{H}}$ are principally down the jet and not across the jet, and so provide similar weightings when we compare sections transverse to the jet axis. We can thus use the fitted $N_{\mathrm{H}}$ and spectral index for our transverse sections of the jet as proxies of systematic trends in the spectra. Appreciable cross-jet differences could then allow us to conclude that there are real variations in the X-ray spectrum across the jet. Selfconsistency tests (see 3 ) verify this assertion. We also find, through comparison with the spectra of individual knots and sections of diffuse emission along the jet (Hardcastle et al. 2007), that any bias in spectral slope in our analysis is small.

In the following, spectral index $\alpha$ (one less than X-ray photon index) is defined in the sense that flux density is proportional to $\nu^{-\alpha}$.

\section{RESULTS}

Our spectral results are summarized in Table 1, and we refer to them here by row number. The quoted uncertainties are $90 \%$ for two interesting parameters, so where pairs of rows disagree in both parameters there is at most a $1 \%$ probability of agreement by chance. Uncertainties in $\alpha$ and $N_{\mathrm{H}}$ are correlated, and so the $\chi^{2}$ contours must be examined to assess 
TABLE 1

Chandra FITS TO A SINGLE-COMPONENT POWER LAW

\begin{tabular}{clccccc}
\hline \hline Row & \multicolumn{1}{c}{ Component/ } & region $\%$ counts & region $\%$ area & $\alpha^{\mathrm{a}}$ & $N_{\mathrm{H}}{ }^{\mathrm{a}}\left(10^{21} \mathrm{~cm}^{-2}\right)$ & $\chi_{\mathrm{min}}^{2} / \mathrm{dof}$ \\
\hline 1 & Spine & 100 & 100 & $0.83_{-0.04}^{+0.05}$ & $1.54_{-0.17}^{+0.18}$ & $961 / 895$ \\
2 & Sheath & 100 & 100 & $0.97_{-0.07}^{+0.08}$ & $1.40 \pm 0.28$ & $527 / 512$ \\
3 & NW sheath & 100 & 100 & $0.98_{-0.11}^{+0.12}$ & $1.47_{-0.44}^{+0.46}$ & $318 / 277$ \\
4 & SE sheath & 100 & 100 & $0.99 \pm 0.11$ & $1.51_{-0.42}^{+0.45}$ & $295 / 282$ \\
5 & Spine knots & 64 & 25 & $0.60 \pm 0.06$ & $1.77_{-0.28}^{+0.29}$ & $602 / 657$ \\
6 & Sheath knots & 49 & 17 & $0.73_{-0.11}^{+0.12}$ & $1.12_{-1.06}^{+0.53}$ & $244 / 254$ \\
7 & Spine diffuse & 36 & 75 & $1.24 \pm 0.10$ & $1.79_{-0.34}^{+0.37}$ & $471 / 407$ \\
8 & Sheath diffuse & 51 & 83 & $1.20 \pm 0.14$ & $1.82_{-0.50}^{+0.51}$ & $338 / 308$ \\
9 & Inner spine knots & 77 & 47 & $0.63 \pm 0.08$ & $2.34_{-0.42}^{+0.44}$ & $474 / 520$ \\
10 & Inner sheath knots & 61 & 33 & $0.94_{-0.18}^{+0.17}$ & $1.64_{-0.76}^{+0.80}$ & $221 / 185$ \\
11 & Outer spine knots & 34 & 18 & $0.98_{-0.18}^{+0.17}$ & $0.09_{-0.06}^{+1.48}$ & $209 / 207$ \\
12 & Outer sheath knots & 27 & 13 & $0.80_{-0.40}^{+0.30}$ & $0.05_{-0.05}^{+1.90}$ & $60 / 71$ \\
13 & Inner spine diffuse & 23 & 53 & $0.96_{-0.17}^{+0.16}$ & $2.85_{-0.88}^{+0.83}$ & $182 / 168$ \\
14 & Inner sheath diffuse & 39 & 67 & $0.90_{-0.22}^{+0.25}$ & $2.73_{-1.16}^{+1.27}$ & $136 / 121$ \\
15 & Outer spine diffuse & 66 & 82 & $1.35_{-0.11}^{+0.12}$ & $1.46_{-0.36}^{+0.40}$ & $318 / 336$ \\
16 & Outer sheath diffuse & 73 & 87 & $1.33_{-0.17}^{+0.19}$ & $1.41_{-0.59}^{+0.63}$ & $191 / 191$ \\
17 & "Bright" spine knots & 88 & 57 & $0.74_{-0.10}^{+0.11}$ & $1.36_{-0.48}^{+0.51}$ & $229 / 249$ \\
18 & "Bright" sheath knots & 82 & 60 & $0.89_{-0.19}^{+0.23}$ & $1.12_{-0.90}^{+0.96}$ & $147 / 124$ \\
\hline
\end{tabular}

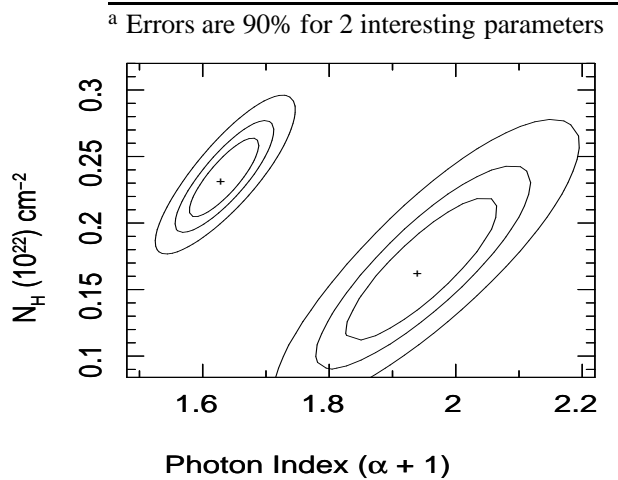

FIG. 3.- Confidence contours $(1 \sigma, 90 \%$ and $99 \%$ for two interesting parameters) showing a significant difference between the spine inner knots (left contours) and sheath inner knots (right contours): rows $9 \& 10$ of Table 1 The $y$ axis begins at Galactic $N_{\mathrm{H}}$.

fully the spectral disagreement. We show such plots only for the comparisons of most interest (Figs. 3 \& 4).

We see (Table 1 rows $1 \& 2$ ) that the sheath spectrum is steeper than the spine. Further investigation allows us to find the origin of this result. The NW and SE regions of the sheath (rows $3 \& 4$ ) give excellent agreement in $\alpha$ and $N_{\mathrm{H}}$, indicating that it is unlikely that the result is from isolated features, and confirming the absence of strong gradients in $N_{\mathrm{H}}$ transverse to the jet. Separating the knot and diffuse emission in the spine and sheath (rows 5 to 8 ) shows that while the knots have flatter spectra than the diffuse emission, the steeper spectrum in the sheath is caused solely by differences in the knots. In the twodimensional plane of $\alpha$ and $N_{\mathrm{H}}$, rows 5 and 6 disagree.

Having established that the knots in the sheath have a steeper spectrum than in the spine, we checked the inner and outer regions to see if there is any trend with distance from the core. Comparing rows 9 and 10 with 11 and 12, we see the expected preference for a higher $N_{\mathrm{H}}$ in the inner regions, but more interestingly we see that it is only for the knots in the inner regions that the spine and sheath spectra differ (Fig. 3). The diffuse emission, in contrast, shows remarkable spectral agreement between the sheath and spine in the inner region (rows $13 \& 14$ ). To check that the contour separation

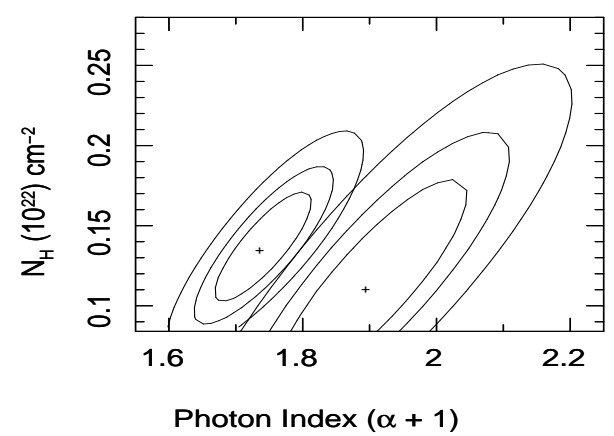

FIG. 4.- Same as Fig. 3 for the bright region spine knots (left contours) and sheath knots (right contours): rows $17 \& 18$ of Table 1

in Figure 3 is causal rather than statistical we have drawn random sets of the required size from the modelled underlying spectral-index distribution deduced from all knots in the inner region. The average spectral index has an error on the mean of \pm 0.07 for knots matching in number those in the inner spine, and \pm 0.11 for the inner sheath. The observed spectral-index difference, $\Delta \alpha=0.31$ (Fig. 3), therefore significantly exceeds the difference expected from random subsamples of the distribution.

Since the spine inner jet knots tend to lie in regions of higher $N_{\mathrm{H}}$ than the sheath inner knots (Figs 2 and 3), we have repeated the analysis only for the "bright" region, marked in blue on Figure 2] This region is beyond the main dust lanes that cross the jet (e.g., fig. 1 of Hardcastle et al. 2007). Results are consistent with sheath knots having steeper spectra than spine knots (Fig. 4 and rows $17 \& 18$ ). Simulations show that the bias $(\S 2)$ on the absolute value of mean spectral index from the $N_{\mathrm{H}}$ distribution over the inner jet is $\sim 0.05$.

We investigated the possibility that the spectral flattening in the inner spine might result from the inner knots being brighter, if brightness is associated with particle-acceleration efficiency. Figure 5 shows the knot surface brightness and hardness across the inner jet. The bin-width is 1 degree in position angle, which corresponds roughly to the HPD at an 

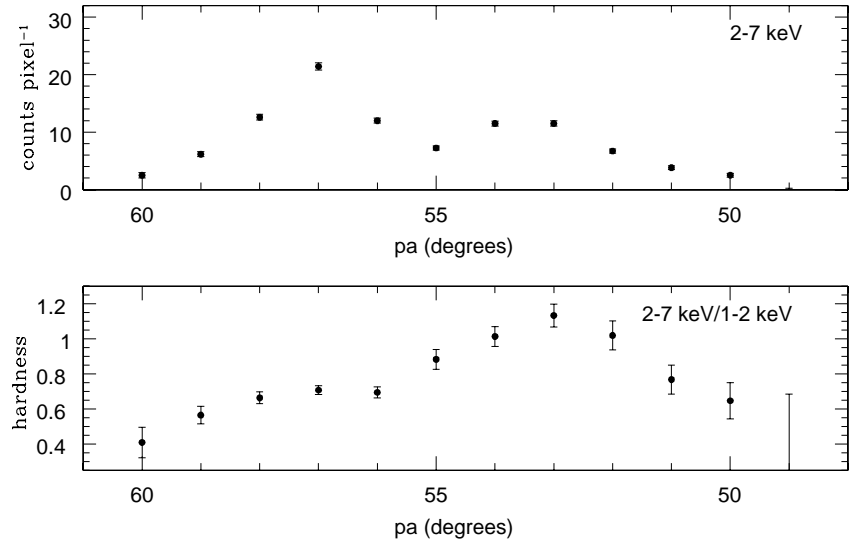

FIG. 5.- Surface-brightness profiles with position angle of the knot emission from the inner jet in a hard energy band (upper panels) with the hardness ratio below. The X-ray emission is softer towards the jet edges $\left(\chi^{2}=116\right.$ for $11 \mathrm{dof}$ for a fit to constant hardness). There is no trend for regions of higher integrated knot surface brightness to show harder spectra.

average jet-axis distance, and so the points are not highly correlated. The hardness ratio shows the decrease with increasing distance from the jet axis that is anticipated from our spectral results, with a high degree of confidence that the hardness is not constant. The gradual decrease of hardness with distance from the jet axis shows that our finding of a spectral difference in the spine and sheath regions was not dictated by the $a$ priori definition of the region boundaries.

Perhaps surprisingly, a comparison of the panels of Figure 5 shows that there is no trend for regions of higher integrated knot surface brightness to be harder in spectrum. We have made plots similar to Figure 5 for the inner and outer diffuse emission, and the outer knot emission. The surface-brightness profiles of diffuse emission show some lumpiness, suggesting that some of what we call diffuse emission is composed of lower-surface-brightness knots, but all three hardness-ratio profiles are consistent with being constant across the jet $\left(\chi^{2}\right.$ values of 10.5, 7.9, and 14.8 for 11 degrees of freedom (dof), as compared with $\chi^{2}=116$ in Fig. 5).

\section{CONCLUSIONS}

If the knots in Cen A were predominantly a surface feature in a shear layer between the spine and sheath, as we suggested might be the case for NGC 315, one would expect the knot Xray spectra to be similar across the jet. This is not what is seen. In the inner jet, between $21^{\prime \prime}$ and $66^{\prime \prime}$ from the core, we have observed in a statistical sense that the X-ray knot spectra steepen farther from the jet axis. No such trend is seen in the more diffuse X-ray emission. The results suggest that knots are distributed throughout the jet volume, with a trend towards steeper spectra in the outer regions. The trend will then be underestimated in our results because of projection effects.

Although the spectral trends disfavor knot confinement in a shear layer, they imply that the electron energy distributions in knots towards the center and edge of the jet are different. Electrons with sufficient energy to emit synchrotron $\mathrm{X}$-rays must be accelerated locally to the knots, since their energy loss timescale is short compared with any dynamical timescale associated with fluid motion. Thus the conditions for particle acceleration differ across the jet. The knots might arise from intruders from outside travelling at speeds close to the stellar velocity dispersion, or internal flow irregularities which are most likely to arise in the central part of the flow and might be expected to move quickly towards the edge of the jet under the influence of circulation in the shear layer. In either case a transverse velocity structure might cause the strength of shocks near the knots to vary across the jet, and since the kinetic energy density of the flow should be higher nearer the jet axis, we might also expect velocity irregularities to generate stronger turbulent cascades near the axis than near the jet edge, with consequent efficient particle acceleration (Manolakou et al. 1999) and a flatter on-axis X-ray spectrum.

As the jet expands, the lower on-axis flow velocity, as suggested by the less collimated appearance of the jet further from the core, would then imply less X-ray spectral difference between sheath and spine, consistent with our results. Indeed, a spectral steepening between the inner and outer regions in diffuse $\mathrm{X}$-ray emission may relate to such a decline in flow speed along the jet (rows 13 to 16 of Table 1 ).

It is interesting to ask whether it will be possible to detect knot migration in Cen A. Expansion of the jet along its axis, with apparent motions of about $0.5 c$, has been measured in radio data from the Very Large Array over 10 years (Hardcastle et al. 2003b), but lateral motion is more difficult to measure. It has been detected in the radio in M 87 (Biretta et al. 1995). We speculate that transverse motions should be present in the knots and are potentially detectable if knots are flow irregularities, although future observations may be required.

These new observations of Cen A have permitted the first $\mathrm{X}$-ray spectral study of a radio jet in the transverse direction, separating knotty and more diffuse emission. We have found the striking result that where the X-ray emission is brightest, at $\sim 0.4$ to $1.2 \mathrm{kpc}$ from the core, the spectrum in the knotty emission is steeper at larger distances from the jet axis. We speculate that this is the result of lateral knot migration.

This work was partially supported by NASA grant GO7$8105 \mathrm{X}$. We thank the referee for constructive comments and MJH thanks the Royal Society for support. Facilities: CXO

\section{REFERENCES}

Biretta, J.A., Zhou, F., \& Owen, F.N. 1995, ApJ, 447, 582

Canvin, J.R., Laing, R.A., Bridle, A.H., \& Cotton, W.D. 2005, MNRAS, 363, 1223

Chiaberge, M., Celotti, A., Capetti, A., \& Ghisellini, G. 2000, A\&A, 358, 104

Dickey, J.M., \& Lockman, F.J. 1990, ARA\&A, 28, 215

Ferrarese, L., Mould, J.R., Stetson, P.B., Tonry, J.L., Blakeslee, J.P., Ajhar, E.A. 2007, ApJ, 654, 186

Hardcastle M.J., Worrall, D.M., Birkinshaw, M., \& Canosa, C.M. 2003a, MNRAS, 338, 176

Hardcastle M.J., Worrall, D.M., Kraft R.P., Forman, W.R., Jones, C., \& Murray, S.S. 2003b, ApJ, 593, 169

Hardcastle, M.J. et al. 2007, ApJ, 670, L81

Jordán, A. et al. 2007, ApJ, in press (arXiv/0711.2303)

Kataoka, J., Stawarz, L., Aharonian, F., Takahara, F., Ostrowski, M. \& Edwards, P.G. 2006, ApJ, 641, 158
Kraft, R.P., Forman, W.R., Jones, C., Murray, S.S., Hardcastle, M.J. \& Worrall, D.M. 2002, ApJ, 569, 54

Laing, R.A. 1996, in ASP Conf. Ser. 100, Energy transport in radio galaxies and quasars, ed. P.E. Hardee,A.H. Bridle \& J.A. Zensus (San Francisco: ASP), 241

Laing, R.A., \& Bridle, A.H. 2002, MNRAS, 336, 1161

Manolakou, K., Anastasiadis, A., \& Vlahos, L. 1999, å, 345, 653

Schwartz, D.A. et al. 2006, ApJ, 640, 592

Worrall D.M., Birkinshaw, M. \& Hardcastle, M.J. 2001, MNRAS, 326, L7

Worrall D.M., Birkinshaw, M., Laing, R.A., Cotton, W.D., Bridle,

A.H. 2007, MNRAS, 380, 2 